\title{
Formation of hippocampal mHTT aggregates leads to impaired spatial memory, hippocampal activation and adult neurogenesis
}

Schwab $\mathrm{LC}^{\dagger^{\star}}$, Richetin $\mathrm{K}^{\dagger}$, Barker RA**, Deglon $\mathrm{N}^{\star *}$

${ }^{\dagger}$ Equal first author, * Corresponding author. ${ }^{* *}$ Equal last authors

Keywords: Adult neurogenesis, memory, neural progenitor cells, dentate gyrus, Huntingtin, lentiviral vectors.

\section{Acknowledgements}

The authors would like to thank M. Pachoud, C. Pythoud and M.Rey for their technical support and Dr Fulvio Magara at Lausanne for his assistance with the behavioural testing. This study was supported by a BBSRC/GSK CASE Studentship awarded to L. Schwab, the synapsis Foundation fellowship awarded to K. Richetin and Swiss National Science Foundation grant 31003A_140945.

\section{Contributors}

LS designed the research study, acquired the data and wrote the manuscript. KR designed the research study, acquired the data and wrote the manuscript. RAB designed the research study and critically revised the manuscript. ND designed the research study and critically revised the manuscript. 


\section{Abstract}

Huntington's disease (HD) is a genetic neurodegenerative disorder characterized by a triad of motor, psychiatric and cognitive deficits with the latter classically attributed to disruption of fronto-striatal circuits. However, emerging evidence suggests that some of the cognitive deficits in HD may have their origin in other structures including the hippocampus.

Hippocampal abnormalities have been reported in HD mouse models particularly in terms of performance on the Morris Water Maze. However, in these animals, it is difficult to be certain whether the spatial memory deficits are due to local pathology within this structure or their poor mobility and motivation. Thus, a better model of hippocampal dysfunction in HD is needed especially given that we have previously shown that patients with HD have hippocampal-related problems from the very earliest stages of disease. In this study, our aim was therefore to understand the cellular and behavioural consequences of local overexpression of mutant huntingtin (mHTT) in the hippocampus of adult mice.

We found that a targeted injection of a lentivirus, encoding an N-terminal of mHTT with 82 CAG repeats, into the murine hippocampus led to the focal formation of $\mathrm{mHTT}$ aggregates, long-term spatial memory impairments with decreased neurogenesis and expression of the immediate early gene c-fos.

This study has therefore shown for the first time that local expression of mHTT in the dentate gyrus has deleterious effects, including its neurogenic capacity, with functional behavioural consequences, which fits well with recent data on hippocampal deficits seen in patients with HD. 


\section{Introduction}

Huntington's disease is an autosomal dominant neurodegenerative disorder characterized by a triad of motor, psychiatric and cognitive deficits with the latter classically being attributed to disruption of fronto-striatal networks. However, emerging evidence suggests that some of the cognitive deficits in HD may take their origin from other structures and networks including the hippocampus. Although the majority of the work conducted on mice models of HD has focused on the striatal and cortical aspects of disease, hippocampal problems have been reported as well as in HD patients (Lione et al. 1999; Giralt et al. 2011; Giralt et al. 2012; Simpson et al. 2011; Gil et al. 2005; Lazic et al. 2004; Phillips et al. 2005; Grote et al. 2005; Begeti et al. 2016). These problems include difficulties with spatial memory (Begeti et al. 2016; Lione et al. 1999; Lüesse et al. 2001; Kung et al. 2007; Murphy et al. 2000), which fits well with the findings that hippocampal synaptic function and plasticity is affected early on in animal models of HD in association with hippocampal nuclear and neuritic mHTT inclusions (Murphy et al. 2000; Lione et al. 1999; Simpson et al. 2011; Gil et al. 2005; Lazic et al. 2004; Phillips et al. 2005; Kolodziejczyk et al. 2014). In addition, studies on transgenic HD mice have also shown that adult hippocampal neurogenesis is also affected, particularly the proliferation and maturation of new-born neurons (Simpson et al. 2011; Gil et al. 2005; Lazic et al. 2004; Phillips et al. 2005; Grote et al. 2005). As new-born hippocampal neurons are hyper-excitable and contribute to the increased plasticity of the hippocampus (Deng et al. 2010), it is therefore possible that this too could contribute to some of these cognitive impairments.

However, to date, it is not well understood whether spatial memory impairments are due to this local hippocampal pathology or the result of $\mathrm{mHtt}$ expression at other sites linked to the hippocampal complex. In addition, it is unclear whether these mnemonic deficits are actually caused by motor deficits and/or a lack of motivation, which is a common early feature in both HD patients and mouse models of disease.

It is now accepted that adult neurogenesis is part of the mechanisms involved in hippocampal plasticity and play an important role in the process of spatial learning and pattern separation (Clelland et al. 2009; Doetsch \& Hen 2005; Aimone et al. 2011). Moreover, concurrent alterations in adult neurogenesis and spatial memory are observed in neurodegenerative diseases such as Parkinson's disease, Huntington's disease and Alzheimer's disease (Winner et al. 2011). In the case of HD, studies on transgenic animal models have shown that adult hippocampal neurogenesis is strongly affected, particularly the proliferation and maturation of new-born neurons (Simpson et al. 2011; Gil et al. 2005; Lazic et al. 2004; Phillips et al. 2005; Grote et al. 2005). As new-born hippocampal neurons 
are hyper-excitable and increase considerably the hippocampus' plasticity (Deng et al. 2010), it is therefore possible to speculate that if compromised, adult hippocampal neurogenesis might play a role in some of the cognitive impairments observed in HD.

In this study, we therefore sought to explicitly investigate the neural basis of hippocampaldependent cognitive deficits in HD by selectively expressing mHTT in the hippocampus. Lentiviral and adeno-associated viral vectors have been previously used to deliver the $m H T T$ gene to specific brain regions experimentally, mainly targeting the striatum but also the hypothalamus and cortex where they have been shown to induce neuropathological and behavioural effects (DiFiglia et al. 2007; Hult et al. 2011; de Almeida et al. 2002; Palfi et al. 2007). In the present study, we used a lentiviral vector to express the first 171 amino acids of mHTT with 82 CAG repeats (LV-mHTT) in the dorsal dentate gyrus (DG) of the murine hippocampus and describe the pathological and behavioural consequences of this.

We found that focal expression of $\mathrm{mHTT}$ in the DG leads to local pathology with the appearance of $\mathrm{mHTT}$ aggregates, long-term spatial memory impairments as well as changes in hippocampal activity, including a focal decrease in c-Fos positive cells and neurogenesis. 


\section{Results}

\section{Injection of LV-mHTT leads to the formation of HTT aggregates in the dentate gyrus}

LV-mHTT was used to investigate the effects of a focal overexpression of mHTT in the dentate gyrus (DG) of the hippocampus. The vector, overexpressing a $\mathrm{N}$-terminal fragment (171 amino acids) of human HTT with a glutamine tract of 82 glutamines, was injected bilaterally into the mouse DG. Twelve weeks following injection, neuronal inclusions (Figure 1A) were detected in the hippocampus using the EM48 antibody, which recognizes the aggregated form of HTT (Figure 1B). Mice which were not injected (NI), and those injected with a lentiviral vector expressing only green fluorescent protein (LV-GFP), were devoid of such inclusions and served as controls (Figure 1B). Topographical observations revealed that the aggregates were mostly present in the hilus and subgranular layer with some spread into the granular cell and molecular layers. No aggregates were observed outside of the hippocampus (Figure S1). Neither injections of LV with transgene expression (GFP or mHTT) affected granule cell layer volume $(F(2,21)=0.66, p=0.53$, Figure $1 C)$. In addition, we observed no significant reactive astrocytosis in any groups (GFAP density: $F(2,34)=1.98$, $p=0.15$, Figure 1B\&D) although, there was an increase in reactive microglia (lba1 density: $F$ $(2,31)=9.59, P<0.0005$, Figure 1B-E) although this was the same in both the LV-GFP and LV-mHTT treated animals compared to the $\mathrm{NI}$ mice. As hippocampal function can be affected by local inflammation (Ekdahl et al. 2003), subsequent analyses were performed using GFP mice for comparison to control for the microglia induced activation associated with the LV injection. 


\section{Figure 1}

\section{A}

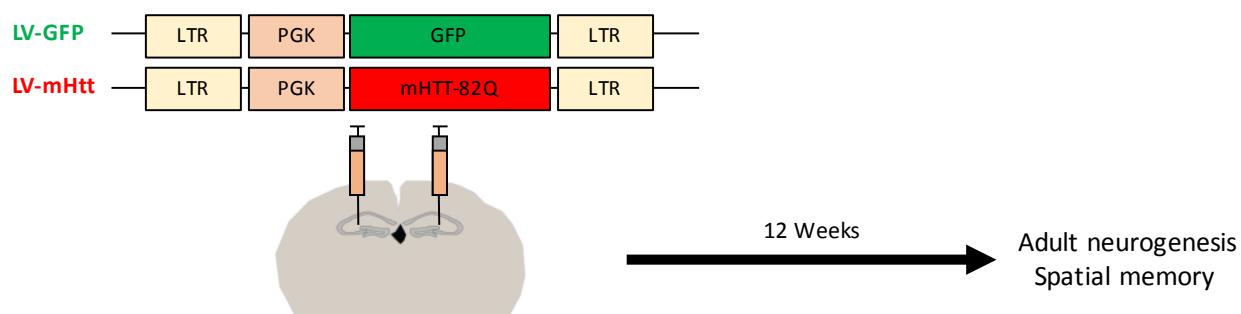

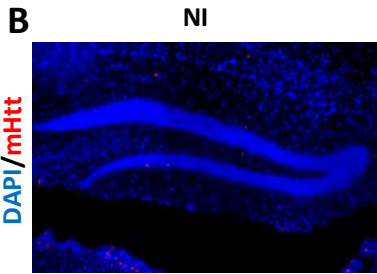
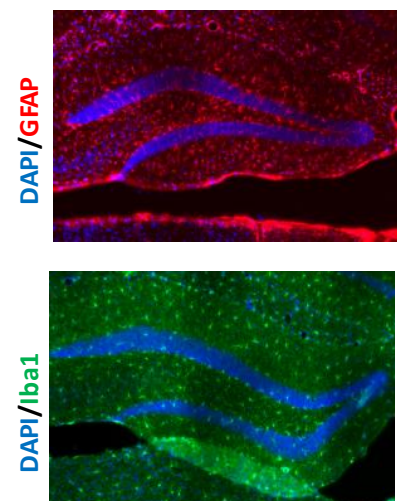

LV-GFP
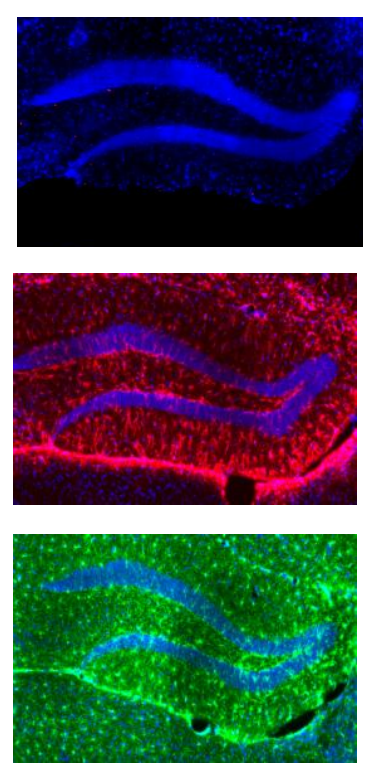

LV-mhtt
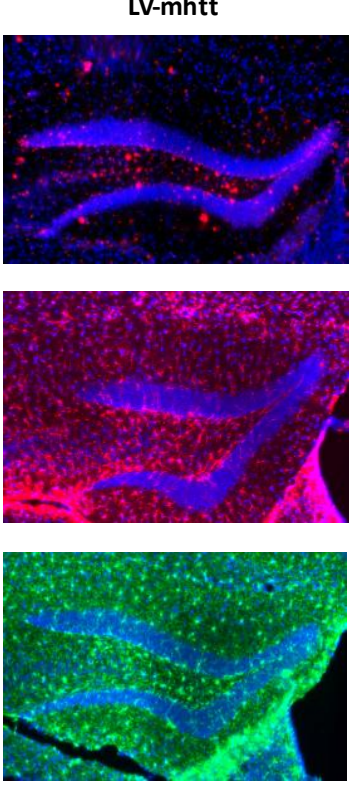

C

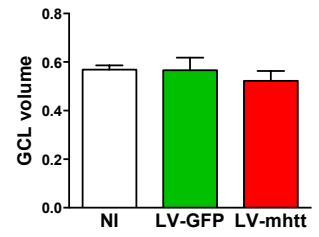

D

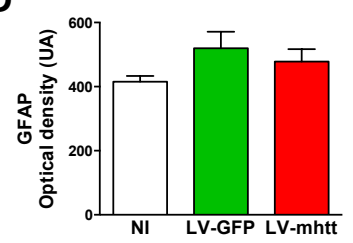

E

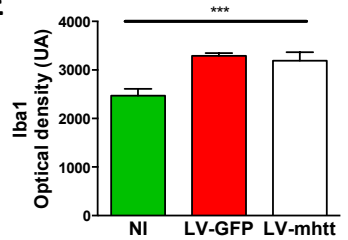

Figure 1: Formation of local $\mathrm{mHTT}$ aggregates after injection of the LV-mHTT into the dentate

gyrus of mice. (A) Lentiviral constructs and experimental design: adult mice were injected with lentiviral vectors encoding GFP (LV-GFP) or a human N-terminal fragment of HTT with 82 CAG repeats (LV-mHTT) in the dentate gyrus using stereotaxic surgery. The effect of the injection on the adult hippocampus was analysed 12 weeks post-injection; (B) Representative images of EM48 staining, GFAP staining (middle panel) and lba1 staining (bottom panel) in non-injected mice (NI: left column) and mice injected with GFP (LV-GFP: middle column) and mHTT (LV-mHTT: right column); (C) Injections of GFP or mHTT did not affect the GCL volume in any groups; (D) There was no significant increase in GFAP optical density in the injected groups compared to the non-injected mice. (E) However, there was a significant increase in reactive microglia as revealed using lba1 optical density measurements, but this was the same for both LV-GFP and LV-mHTT mice. Quantitative data are means \pm SEM. Analyses were performed using One-way ANOVA: ${ }^{* * *} p<0.0005$. Scale bar: $200 \mu \mathrm{m}$. 


\section{Hippocampal mHTT aggregates lead to impairments in long-term spatial memory}

We examined whether the focal overexpression of $\mathrm{mHTT}$ in the DG had an impact on spatial learning and memory in a well-validated spatial navigation task- the Morris Water Maze (Figure 2A). To ensure that any changes in performance were not due to locomotion deficits, the velocity of swim was also recorded and no difference was observed between any of the groups (Figure 2B). Both LV-GFP and LV-mHTT injected mice learned to locate the platform following 4 days of training $((F(3,44)=7.59, p=0.0003$, Figure $2 \mathrm{C})$. Spatial memory was assessed during a probe session immediately or 24 hours after the last training trial. LV-GFP and LV-mHTT mice did not show altered short-term memory as they all crossed the target platform preferentially (Figure 2D). However, 24h after the last training trial, LV-mHTT-injected mice did not show any preference for the target annulus compared to other annuli whereas the LV-GFP mice preferentially crossed the target annulus (mHTT $p=0.059$, GFP $p=0.042$, Figure $2 E$ ). This suggests that aggregation of $m H T T$ in the $D G$ does not affect normal learning but does impair long-term spatial memory. 


\section{Figure 2}

A

Hippocampal stereological injections

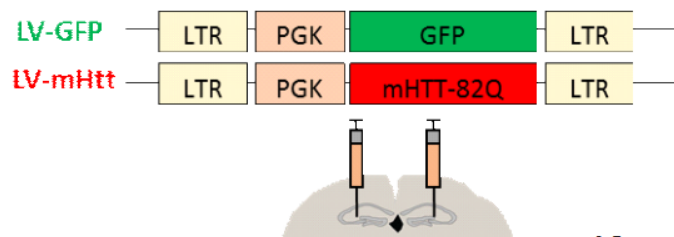

\section{Spatial Morris Water Maze}

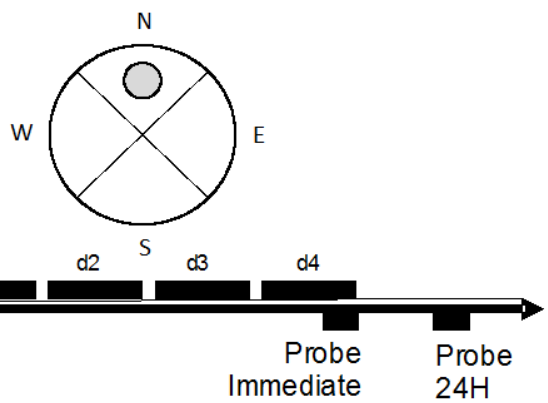

B

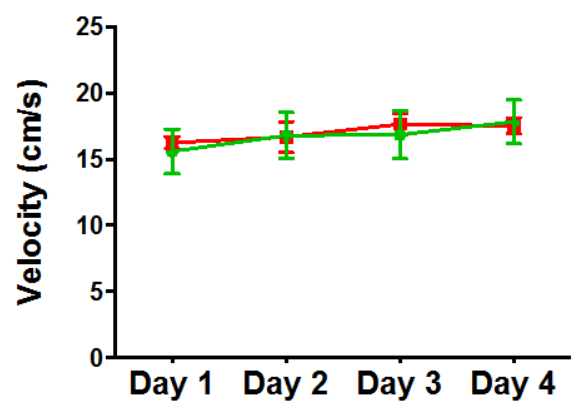

D

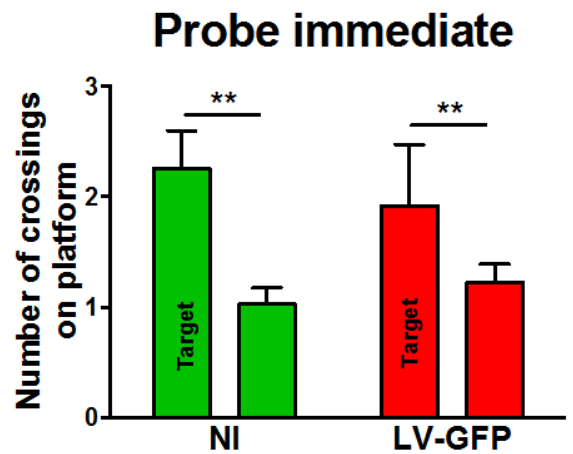

C

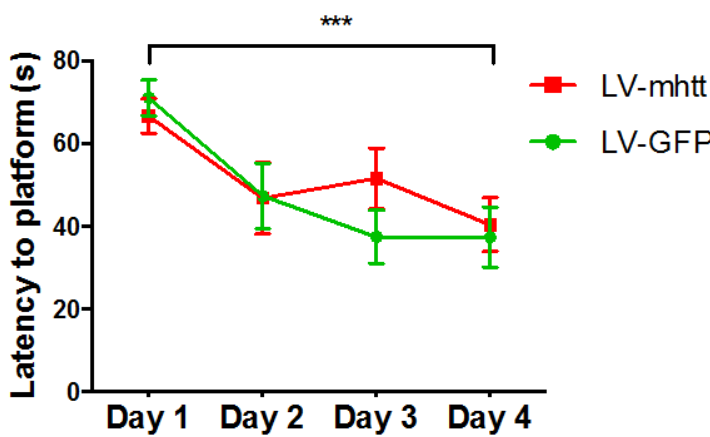

$\mathbf{E}$

\section{Probe 24H}

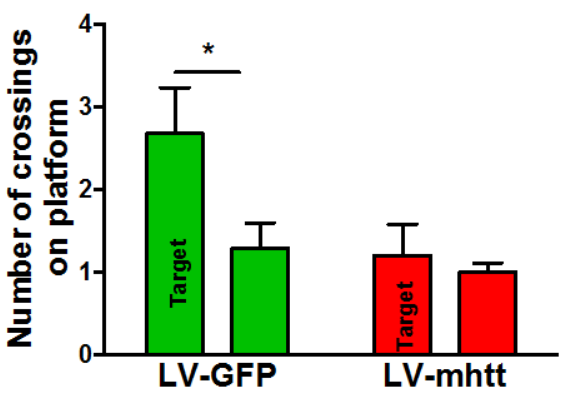

Figure 2: Expression of $\mathrm{mHTT}$ in the dentate gyrus impaired long-term spatial memory using the Morris Water Maze. (A) Lentiviral constructs and experimental design: Mice were injected with LV-GFP or LV-mHTT and spatial learning and memory were evaluated 12 weeks post-injection. (B) Swimming velocity was measured on each training day to ensure that the injection had no effect on motor performance. No differences were seen. (C) The latency to find the hidden platform was measured on each training day to assess learning. Both groups were able to learn the location of the platform as the latency to find the hidden platform significantly decreased over the four training days. (C) Immediately or (D) 24 hours following the last training day, a probe session was performed during which the platform was removed and the number of crossings above the old location of the target platform were measured and compared to the average number of crossings over the other quadrants. There was no increase in the number of crossings above the target platform in the LV-mHTT mice showing that the mice did not remember the location of the platform 24 hours following the last day of training. Quantitative 
data are means \pm SEM. Data were analysed using repeated measures ANOVA and paired t-tests: * $p<0.05 ;{ }^{* *}$ $p<0.005,{ }^{* * *} p<0.0005$.

\section{Hippocampal mHTT aggregates lead to localised altered immediate early gene expression in the hippocampal dentate gyrus.}

Immediate early genes (IEGs) such as Zif268, c-Fos and Arc are rapidly and transiently induced by neuronal activation after spatial memory processing (Kubik et al. 2007; Barry \& Commins 2011). We therefore examined the effect of hippocampal expression of mHTT on the number of c-Fos positive nuclei 90 minutes after the probe test session in the Morris Water Maze task (Figure 3A). We observed that LV-mHTT injected mice had a significant reduction in the number of $c$-Fos positive cells in the granular cell layer of the DG (c-Fos number: $t(13)=3.090 p=0.086$, Figure 3B-C), while the number of $c$-fos positive cells was unchanged in the somatosensory cortex, a region used as a comparative control (Figure3BC). 


\section{Figure 3}

A

Hippocampal stereological injections

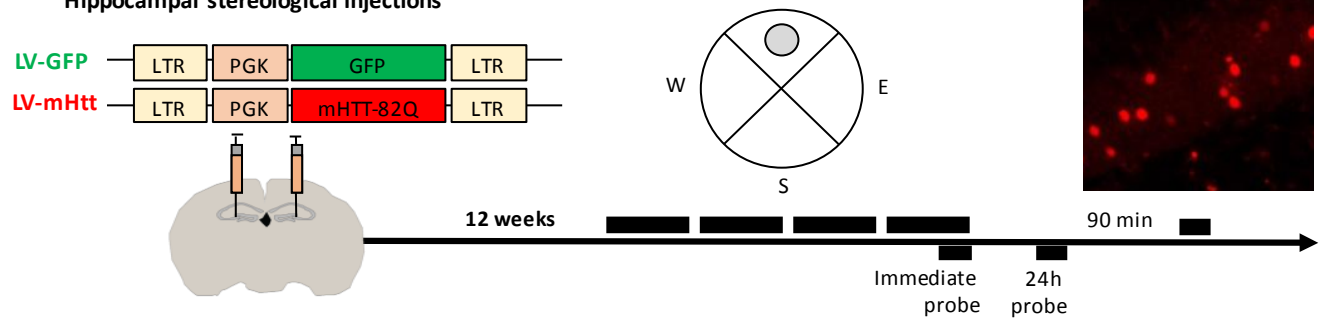

B

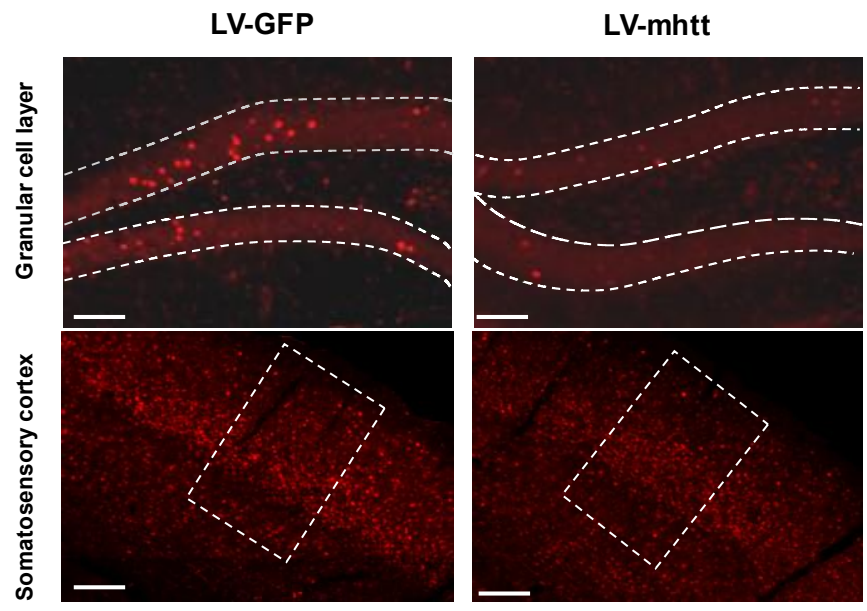

C

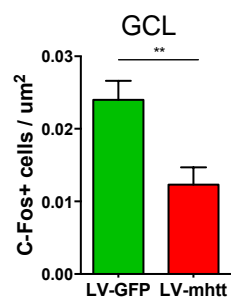

D

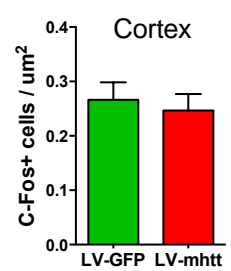

Figure 3: LV-mHTT altered neuronal activation in the adult murine hippocampus as evidenced by changes in the number of $\mathbf{c}$-fos positive cells. (A) Experimental design: Hippocampal c-fos positive cells were evaluated in the dentate gyrus and the cortex of LV-GFP and LV-mHTT mice 12 weeks following viral injection, and 90 minutes after the Morris water maze probe session. (B) Representative images of c-Fos staining in the DG of the hippocampus and the somatosensory cortex (C) Number of c-Fos positive cells per $\mu \mathrm{m}^{2}$ in the DG (upper graph) and the somatosensory cortex (lower graph) 12 weeks after injection of LV-GFP (left panel) and LV-mHTT (right panel). In the DG, LV- $m H T T$ mice displayed a significantly smaller number of c-Fos positive cells compared to LV-GFP mice; however this was not the case in the somatosensory cortex. Quantitative data are means \pm SEM. Data were analysed using independent t-tests: ${ }^{* *} p<0.005$. Scale bar: $50 \mu \mathrm{m}$. 


\section{Local mutant HTT expression leads to a decrease in hippocampal adult neurogenesis}

We then examined the effect mHTT on adult hippocampal neurogenesis, specifically in the DG. We first evaluated the proliferation of new-born cells using ki67 and found that Ki67+ cells were significantly reduced in mHTT mice compared to GFP-injected mice $(t(10)=4.83, p=0.0007$, Figure 4A). We then examined the survival of adult-generated hippocampal cells by quantifying the number of BrdU+ cells in the DG in mice that had been systemically injected with this agent 30 days before sacrifice. We found that mice injected with LV-mHTT had a significant decrease (37\%) in the number of BrdU-positive cells compared to LV-GFP mice $(t(11)=3.472, p=0.005$, Figure 4B). Finally, we characterized the BrdU/DCX and BrdU/NeuN-positive cells and found that mHTT injected mice displayed a decrease in surviving immature (colocalisation BrdU/DCX: $t(6)=2.77, p=0.032$, Figure 4C) and mature neurons (colocalisation BrdU/NeuN: $t(6)=3.26, p=0.017$, Figure 4D) compared to controls. 


\section{Figure 4}
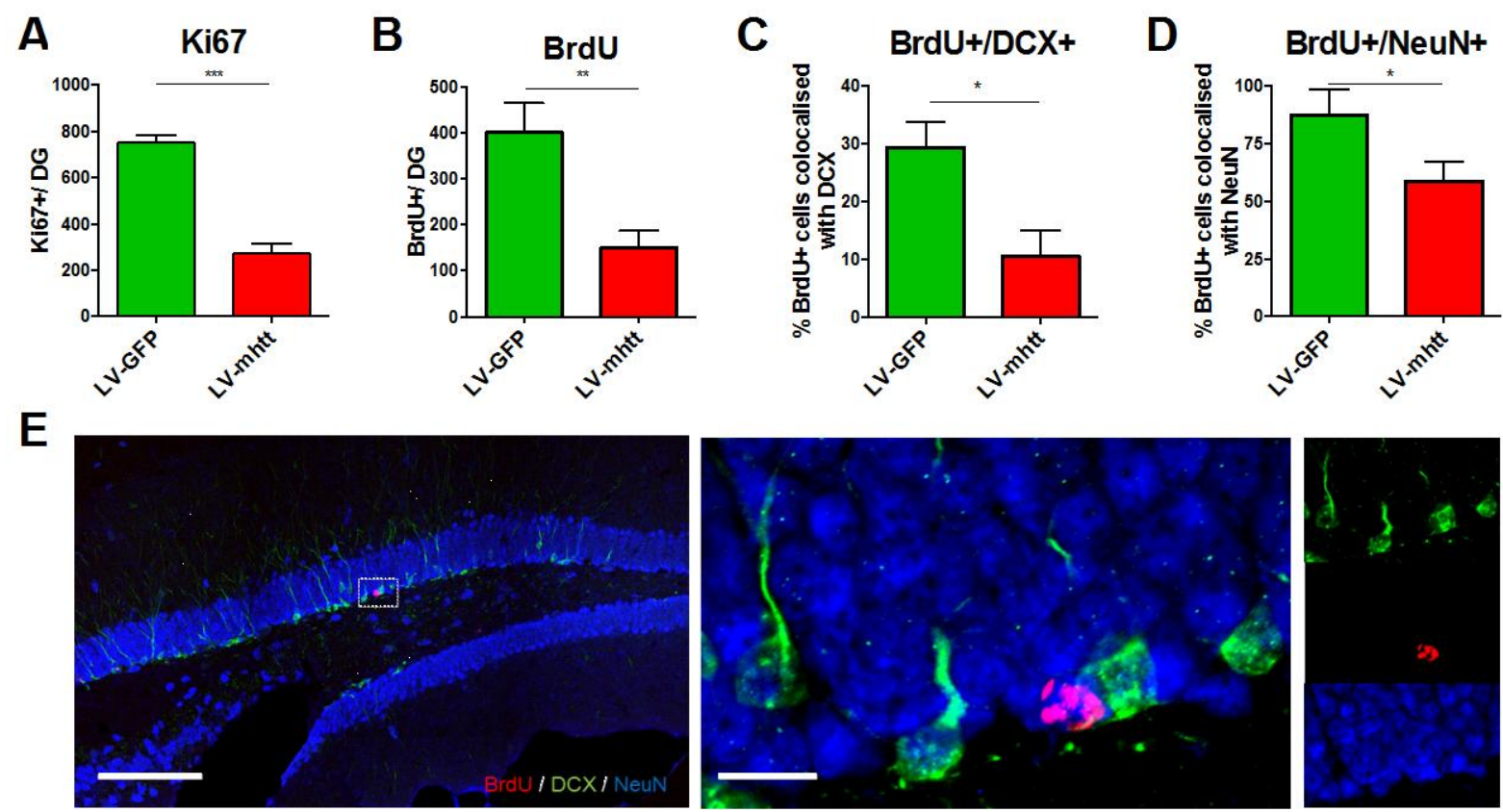

Figure 4: Decrease in proliferation, neuronal survival and maturation following focal injection of LV-mHTT into the DG. (A) Hippocampal cell proliferation was measured in the dentate gyrus of LV-GFP and LV-mHTT mice 12 weeks following viral injection. LV-mHTT mice had a decrease in the number of ki67+ cells compared to LV-GFP mice. (B) All mice received BrdU injections and the number of surviving BrdU+ cells was evaluated 30 days later. LV-mHTT mice displayed a decrease in surviving BrdU+ cells compared to LV-GFP controls. (C) and (D) Percentage of different types of BrdU cells in both LV-mHTT and LV-GFP treated mice. LVmHTT mice displayed a significantly smaller number of BrdU+ cells co-stained with doublecortin (DCX) and the neuronal marker NeuN compared to LV-GFP mice. (E) Representative merged confocal images depicting colocalisation of BrdU with DCX and NeuN. Quantitative data are means \pm SEM. Data were analysed using independent t-tests: ${ }^{*} p<0.05 ;{ }^{* \star} p<0.005,{ }^{* * *} p<0.0005$. Scale bar: $200 \mu \mathrm{m}$ and $10 \mu \mathrm{m}$. 


\section{Discussion}

In this study, we have shown that injection of LV-mHTT specifically into the DG induces local mHTT aggregation, changes in spatial memory and hippocampal plasticity neurogenesis and activation. These results show that $\mathrm{mHTT}$ expression localised to the hippocampus does has effects, which fits with the current animal and emerging human data showing hippocampal problems in HD.

\section{Hippocampal deficits in animal models of HD}

The role of the striatum has been extensively studied in HD while other brain regions have tended to receive less attention such as the hippocampus. However it is known that some of the cognitive dysfunction seen early in the disease as well as possibly some of the depressive behaviours (Spargo et al. 1993; Rosas et al. 2003; van den Bogaard et al. 2011; Lione et al. 1999; Murphy et al. 2000), may have an origin in this structure.

In transgenic animal models of HD, early studies showed there were changes in LTP as well as nuclear inclusions of $\mathrm{mHTT}$ in the hippocampus and the formation of aggregates which correlated with age and reduced synaptic plasticity (Murphy et al. 2000). Pathology in this region was shown early on to be linked to impairments in spatial memory in the Morris Water Maze (Lione et al. 1999). In addition, several studies, including our own, also found that hippocampal neurogenesis in transgenic HD mice was affected and that this also involved neuronal differentiation and proliferation (Simpson et al. 2011; Gil et al. 2005; Lazic et al. 2004; Phillips et al. 2005; Grote et al. 2005; Fedele et al. 2011).

Despite these multiple studies, it still remained unclear whether these deficits (Lione et al. 1999; Brooks et al. 2012; Murphy et al. 2000; Nithianantharajah et al. 2008) could be solely accounted for by localised pathology within the hippocampus, rather than pathology at other sites that are connected to the hippocampus and/or motor and/or motivational problems that have a locus of effect outside this structure (Lione et al. 1999; Lüesse et al. 2001; Kung et al. 2007; Murphy et al. 2000).

In light of this, we sought to target disease-related gene expression using viral vectors, to the hippocampus and study its effects on neurogenesis, plasticity neural activation and behaviour and how this related to any pathology we induced at this site. 


\section{Hippocampal deficits in HD patients}

This is clinically important as well, given that hippocampal pathology is seen in HD patients from early on in the disease course (Spargo et al. 1993; Rosas et al. 2003; van den Bogaard et al. 2011; Lione et al. 1999; Murphy et al. 2000).

Post-mortem and imaging studies with HD patients have demonstrated that there is a reduction in hippocampal volume along with degeneration from the pre-manifest disease stage (Spargo et al. 1993; Rosas et al. 2003; Van Den Bogaard et al. 2011).

While one study published by our group has recently focused on hippocampal-dependent memory in HD patients (Begeti et al. 2016), others studies have investigated the performance of patients on multiple memory tasks which includes hippocampal dependent cognitive processes. In pre-manifest patients, some memory deficits are apparent in patients without obvious clinical features of HD but who are nevertheless predicted to be close to disease onset (Stout et al. 2011) and this includes problems with working memory (Verny et al. 2007) and episodic memory (Montoya et al. 2006; Verny et al. 2007).

In manifest patients, memory is greatly impaired and the range of deficits widens with deficits seen in learning, visuospatial memory (Lange et al. 1995; Lawrence et al. 2000), short-term memory (Ho et al. 2003; Kirkwood SC et al. 2001) and long-term memory including both declarative (Sprengelmeyer et al. 1995) and procedural forms (Butters et al. 1985). Deficits in pattern and spatial recognition memory, spatial learning and working memory have also been consistently shown to be affected in HD (Lange et al. 1995; Brandt et al. 2005; Lawrence et al. 2000; Majerová et al. 2012; Begeti et al. 2016). Ultimately, in HD patients cognitive function declines and a subcortical dementia develops (Zakzanis 1998; Paulsen et al. 1995). Therefore, the documented degeneration and functional abnormalities in the hippocampus may underlie some of the cognitive deficits observed in HD and play a role in disease progression.

\section{The cause of spatial memory impairments in HD}

Although no causal link can be established in our studies, it can be speculated that spatial memory impairments did correlate with reduced neurogenesis and local pathology within this structure. In fact, adult hippocampal neurogenesis has previously been linked to spatial learning and memory with studies showing that dentate granule neurons may be essential for long-term spatial memory in various mazes, including the Morris Water Maze (Clelland et al. 2009; Doetsch \& Hen 2005; Snyder et al. 2005; Winocur et al. 2006; Epp et al. 2013). However, hippocampal-dependent learning is also a key modulator of hippocampal neurogenesis, with tasks such as the Morris Water Maze increasing the number of adult- 
born granule cells (Ambrogini et al. 2000; Epp et al. 2010; Gould et al. 1999; Leuner et al. 2004; Richetin et al. 2015; Petsophonsakul et al. 2017). Therefore, conditions affecting hippocampal neurogenesis are expected to have an impact on overall hippocampal function and vice versa. One way though to look at the modulation of adult neurogenesis by behaviour is through the expression of immediate early genes (IED) a few hours following the cognitive test. The expression of IEDs are known to be closely related to hippocampal neuronal activity following learning and memory tasks and therefore can provide a readout of neuronal activity in this region. We therefore chose to do this and found that c-fos was downregulated in the hippocampus of LV-mHTT mice compared to LV-GFP mice, which was not the case in another region- the somatosensory cortex- unrelated to spatial memory. This suggests therefore that local expression of $\mathrm{mHTT}$ affects hippocampal neuronal activity in addition to neurogenesis. In this respect, it would be interesting to look further at this using electrophysiological approaches and studies of LTP- but these were not undertaken in our experiments.

\section{Conclusions}

This is the first study to look at the effect of local expression of $\mathrm{mHTT}$ in the DG and to show that targeted expression of $\mathrm{mHTT}$ to this structure is sufficient to induce a pathology with aggregate formation and neurogenic alterations associated with spatial memory deficits. Local injection of mHTT may therefore provide a potential method by which to dissect the mechanisms underlying the wide range of psychiatry and cognitive deficits seen in HD, which in turn may have implications for the development of better therapeutic agents including targeted gene silencing approaches that are now entering early phase clinical trials. 


\section{Materials and Methods}

\section{Animals}

Thirty-six adult female C57/BI6 wild type (WT) mice ( $\mathrm{N}=12$ per group) were purchased from Janvier (Le Genest-St-Isle, France). All animals were kept on a normal 12h light/dark cycle and had access to food and water ad libitum. Mice were separated into cages of 4 mice per cage. All procedures were performed in strict accordance with the appropriate Home office project and personal licenses and the Swiss legislation on the care and use of laboratory animals. The animals were housed in a temperature-controlled room $\left(22^{\circ} \mathrm{C} \pm 1^{\circ} \mathrm{C}\right)$ and maintained on a $12 \mathrm{~h}$ light/night cycle. Food and water were available ad libitum. Tissue analyses were performed in Lausanne and in the John van Geest Centre for Brain Repair, University of Cambridge, UK.

\section{Lentiviruses}

The LV-mHTT and LV-GFP vectors used in this study have been previously described (de Almeida et al. 2002). The self-inactivating transfer vectors (SIN) expressing the first 171 amino acids of the HTT protein with 82 CAG repeats or green fluorescent protein (GFP) reporter gene contained a woodchuck hepatitis virus $B$ post-regulatory element (WPRE) and the mouse PGK promoter. The LVs were pseudotyped with the vesicular stomatitis virus glycoprotein G (VSV-G) protein. Viral particles were produced by transfection of human embryonic kidney (HEK) 293T cells (mycoplasma negative, ATCC, LGC Standards GmbH, Germany). Viruses were concentrated by ultracentrifugation and re-suspended in phosphate-buffered saline (dPBS, Gibco, Life Technologies, Zug, Switzerland) with 1\% bovine serum albumin (BSA, Sigma-Aldrich, Buchs, Switzerland). The viral particle content of each batch was determined by a p24 antigen enzyme-linked immunosorbent assay (p24 ELISA, RETROtek; Kampenhout, Belgium). The stocks were stored at $-80^{\circ} \mathrm{C}$ until use. Viral stocks were thawed and re-suspended by pipetting. All viral particles were diluted at 300 $000 \mathrm{ng} / \mathrm{ml}$ in $\mathrm{PBS} / 1 \% \mathrm{BSA}$.

\section{Animals and stereotaxic delivery of viral vectors}

Thirty-six adult C57/BI6 mice (Janvier,Le Genest-St-Isle, France) were used and divided into 3 groups: LV-mHTT injection, LV-GFP injection and no injection ( $\mathrm{NI}$; $\mathrm{N}=12$ per group). Mice were anesthetized by intraperitoneal injection of a mixture of $100 \mathrm{mg} / \mathrm{kg}$ ketamine (Ketasol, Graeub, Bern, Switzerland) and $10 \mathrm{mg} / \mathrm{kg}$ xylazine (Rompun, Bayer Health Care, Uznach, Switzerland). The animals received $2 \mu \mathrm{l}$ of viral vectors bilaterally injected into the dorsal dentate gyrus at the coordinates: $+/-1.5$ lateral to the midline, -2 posterior to bregma, -2.5 
ventral to the dura relative to bregma. The viruses were injected at $0.2 \mu \mathrm{l} / \mathrm{min}$ and the needle was left in place for $5 \mathrm{~min}$. Animals received injection of the analgesic buprenorphine at a dose of $0.1 \mathrm{ml}$ per $100 \mathrm{~g}$ post viral delivery. The skin was sutured with a 6-0 Prolene suture (B-Braun Medical SA, Sempach, Switzerland) and the mice were allowed to recover on a heating mat before returning to their home cages.

\section{BrdU Injection- Survival}

To assess the survival of new-born cells, mice received three intraperitoneal injections of 5bromo-2-deoxyuridine (BrdU) (100 mg/kg in 0.9\% saline; Sigma, St. Louis, MO) four hours apart and were perfused 30 days later.

\section{Spatial learning and memory task}

The Morris Water Maze task was used to study spatial learning and memory. Mice from the three groups were trained to locate a hidden platform using distal cues on four blocks of three trials each day for 4 days. During the training phase, mice were placed in a pool filled with opaque water set at $25^{\circ} \mathrm{C}$. The platform was always hidden in the South East quadrant of the pool but the mice were released at various points around the swimming pool, with the point of release being counterbalanced every day. The inter-trial interval was between 10 and 20 mins. Spatial memory was then assessed during an immediate probe on the $4^{\text {th }}$ day and a probe trial 24 hours after the final training trial, on the $5^{\text {th }}$ day during which the hidden platform is removed. Measures for the training phase included latency to find the platform and velocity of swimming. During the probe tests, time spent in each quadrant and the numbers of crossings over the location of the platform (virtual circle) compared to the mean crossing number of the three other virtual circles in the other 3 quadrants was calculated.

\section{Histological processing}

\section{Tissue preparation}

Two hours following the probe trial, mice were deeply anaesthetized with a sodium pentobarbital overdose, transcardially perfused with $0.1 \mathrm{M}$ phosphate buffer (PBS, $\mathrm{pH}=7.4$ ) followed by $4 \%$ paraformaldehyde (PAF). Brains were removed and post fixed in $4 \% \mathrm{PAF}$ for 24 hours and finally transferred to $0.1 \mathrm{M}$ PBS with $30 \%$ sucrose at $4^{\circ} \mathrm{C}$ for $48-72$ hours. Brains were cut on a freezing microtome into $30 \mu \mathrm{m}$-thick free-floating coronal sections and stored in cryoprotectant at $-20^{\circ} \mathrm{C}$ until use.

\section{Immunohistochemical procedure}


For the immunolabelling, free-floating sections were rinsed in PBS with $0.3 \%$ Triton-X-100 (PBST). For BrdU staining, sections were incubated in $3 \mathrm{~N} \mathrm{HCL}$ for 1 hour to denature DNA and then neutralized using borate buffer $(\mathrm{pH}=8.5)$. Sections were blocked using $2.5 \%$ normal donkey serum for 60 min and incubated overnight in $2.5 \%$ normal donkey serum with rat anti-BrdU antibody (1:500, abcam) overnight at room temperature. Sections were washed twice in PBST before being incubated with 550 donkey anti-rat secondary antibody $(1: 500)$ in PBST and rinsed and mounted on $1 \%$ gelatin coated slides, coverslipped using Fluorosave and stored at $4^{\circ} \mathrm{C}$. Other immunolabelling was done in the same way except without the HCL and borate buffer step. The following primary antibodies were used: anti-mouse EM48 (mHTT, 1/500, Millipore, MAB5374), mouse anti-NeuN (neuronal, 1/200, Millipore, MAB377), rabbit anti-Ki67 (proliferative cells, 1/200, Vector Lab, VP-RM04), goat anti-DCX (immature neurons, 1/200, Santa Cruz, sc-8066), c-fos (immediate early gene, Merck, ABE457), GFAP (astrocyte, 1/500, Dako z0334), Iba1 (microglia, 1/00, Abcam ab5076). Sections were incubated with a combination of secondary antibodies depending on the staining: Alexa 488conjugated donkey anti rabbit (1:250), Alexa-550 donkey anti goat (1:250), Alexa-647 donkey anti mouse (1:250). Only mice with injections at the correct target (determined by localising EM48 and GFP staining) were included in the analysis (NI: $n=12 / 12$; LV-GFP: 7/12; LV-mHTT: 7/12).

\section{Cell counting}

\section{Quantification}

\section{BrdU}

Quantification of BrdU-immunoreactive (BrdU+) cells was conducted as previously described (Krezymon et al. 2013). One in 6 series of sections spaced $180 \mu \mathrm{m}$ apart and spanning the rostro-caudal extent of the dorsal dentate gyrus were used for counting the number of BrdU positive cells. Cells located within the granule cell layer and adjacent subgranular zone, defined as a two cell-body-wide zone along the border between the granule cell layer and the hilus, were counted using a $\times 40$ objective. The corresponding surface area of GCL/SGZ sampled for counting was measured using imageJ. The reference volume was determined as the sum of the traced areas multiplied by the distance between sampled sections $(180 \mu \mathrm{m})$. Density of BrdU + cells was then calculated by dividing the number of labelled cells by the granule cell layer sectional volume. The total number of BrdU+ cells was estimated by multiplying these densities by the reference volume. 
One in twelve series of sections spaced $360 \mu \mathrm{m}$ apart containing the dorsal dentate gyrus was labelled with Ki-67. Positive cells located in the subgranular zone were counted using a x40 and x63 objectives of a Leica confocal microscope.

\section{Quantification of BrdU/DCX/NeuN triple-labelled cells}

The number of mature and immature neurons among the surviving cells labelled with BrdU were counted in 1 in 12 sections. The percentage of cells co-expressing NeuN or DCX was determined manually for each BrdU+ cell using a confocal microscope. The analysis was performed over the whole z-axis using a x100 oil immersion objective.

\section{Iba1 and GFAP optical density}

Glial Fibrillary Acidic Protein (GFAP) and lonized calcium binding adapter molecule 1 (lba1) labelling was quantified by measuring GFAP and Iba1 immunoreactivity density in 1 in 12 sections. The non-specific staining optical density in the dorsal third ventricle was subtracted from the optical density of the specific area to calculate an optical density corresponding to specific GFAP and lba1 activity. Non-specific staining was not significantly modified by injection (data not shown). Images were obtained with a digital camera (3CCD Hitachi HVF202SCL) on a slide scanner microscope (x10 objective, Zeiss axioscan Z1). Integrated optical density (IOD) was measured using image analysis software Zen 2 (blue edition).

\section{Quantification of c-Fos positive cells}

One in twelve series of sections spaced $360 \mu \mathrm{m}$ apart containing the dorsal dentate gyrus and the somatosensory cortex was labelled with c-Fos. Images were obtained with a digital camera (3CCD Hitachi HV-F202SCL) on a slide scanner microscope (x10 objective, Zeiss axioscan Z1). Positive cells located in the region of interest (granular zone of the hippocampus, or somatosensory cortex) were counted using image analysis software Zen 2 (blue edition), and cell density was calculated.

\section{Statistical analysis}

Cell counts were multiplied by 6 or 12 depending on the fraction of sections labelled to get an estimate of the total number of cells per dentate gyrus. Data are expressed as mean +/SEM and were analysed using a one-way ANOVA and Student t-test where appropriate with a significance level set at $p<0.05$. Behavioural analyses were performed using a repeated measures ANOVA and independent t-tests where appropriate. 


\section{References}

Aimone, J.B. et al., 2011. Resolving New Memories: A Critical Look at the Dentate Gyrus, Adult Neurogenesis, and Pattern Separation. Neuron, 70(4), pp.589-596.

de Almeida, L.P. et al., 2002. Lentiviral-mediated delivery of mutant huntingtin in the striatum of rats induces a selective neuropathology modulated by polyglutamine repeat size, huntingtin expression levels, and protein length. The Journal of neuroscience : the official journal of the Society for Neuroscience, 22(9), pp.3473-83. Available at: http://www.ncbi.nlm.nih.gov/pubmed/11978824.

Ambrogini, P. et al., 2000. Spatial learning affects immature granule cell survival in adult rat dentate gyrus. Neuroscience letters, 286(1), pp.21-4. Available at: http://www.ncbi.nlm.nih.gov/pubmed/10822143 [Accessed June 6, 2016].

Barry, D.N. \& Commins, S., 2011. Imaging spatial learning in the brain using immediate early genes: insights, opportunities and limitations. Reviews in the Neurosciences, 22(2), pp.131-142.

Begeti, F. et al., 2016. Hippocampal dysfunction defines disease onset in Huntington's disease. Journal of neurology, neurosurgery, and psychiatry. Available at: http://www.ncbi.nlm.nih.gov/pubmed/26833174 [Accessed March 29, 2016].

van den Bogaard, S.J.A. et al., 2011. Shape analysis of subcortical nuclei in Huntington's disease, global versus local atrophy--results from the TRACK-HD study. Journal of the neurological sciences, 307(1-2), pp.60-8. Available at: http://www.ncbi.nlm.nih.gov/pubmed/21624624 [Accessed April 22, 2016].

Van Den Bogaard, S.J.A. et al., 2011. Early atrophy of pallidum and accumbens nucleus in Huntington's disease. Journal of Neurology, 258(3), pp.412-420. Available at: http://www.ncbi.nlm.nih.gov/pubmed/20936300 [Accessed April 22, 2016].

Brandt, J. et al., 2005. Differential impairment of spatial location memory in Huntington's disease. Journal of neurology, neurosurgery, and psychiatry, 76(11), pp.1516-9. Available at:

http://www.pubmedcentral.nih.gov/articlerender.fcgi?artid=1739409\&tool=pmcentrez\&re ndertype=abstract [Accessed May 8, 2014].

Butters, N. et al., 1985. Memory disorders associated with huntington's disease: Verbal recall, verbal recognition and procedural memory. Neuropsychologia, 23(6), pp.729_ 743.

Clelland, C.D. et al., 2009. A functional role for adult hippocampal neurogenesis in spatial pattern separation. Science (New York, N.Y.), 325(5937), pp.210-3. Available at: http://www.pubmedcentral.nih.gov/articlerender.fcgi?artid=2997634\&tool=pmcentrez\&re 
ndertype $=$ abstract [Accessed October 18, 2013]

Deng, W., Aimone, J.B. \& Gage, F.H., 2010. New neurons and new memories: how does adult hippocampal neurogenesis affect learning and memory? Nature reviews. Neuroscience, 11(5), pp.339-50. Available at:

http://www.pubmedcentral.nih.gov/articlerender.fcgi?artid=2886712\&tool=pmcentrez\&re ndertype $=$ abstract [Accessed May 26, 2014].

DiFiglia, M. et al., 2007. Therapeutic silencing of mutant huntingtin with siRNA attenuates striatal and cortical neuropathology and behavioral deficits. Proceedings of the National Academy of Sciences, 104(43), pp.17204-17209. Available at: http://www.pnas.org/cgi/doi/10.1073/pnas.0708285104 [Accessed May 13, 2016].

Doetsch, F. \& Hen, R., 2005. Young and excitable: the function of new neurons in the adult mammalian brain. Current opinion in neurobiology, 15(1), pp.121-8. Available at: http://www.ncbi.nlm.nih.gov/pubmed/15721754 [Accessed October 17, 2013].

Ekdahl, C.T. et al., 2003. Inflammation is detrimental for neurogenesis in adult brain. Proceedings of the National Academy of Sciences of the United States of America, 100(23), pp.13632-7. Available at: http://www.ncbi.nlm.nih.gov/pubmed/14581618 [Accessed July 28, 2016].

Epp, J.R., Chow, C. \& Galea, L. a M., 2013. Hippocampus-dependent learning influences hippocampal neurogenesis. Frontiers in neuroscience, 7(April), p.57. Available at: http://www.pubmedcentral.nih.gov/articlerender.fcgi?artid=3627134\&tool=pmcentrez\&re ndertype $=$ abstract [Accessed January 9, 2014].

Epp, J.R., Haack, A.K. \& Galea, L.A.M., 2010. Task difficulty in the Morris water task influences the survival of new neurons in the dentate gyrus. Hippocampus, 20(7), pp.866-76. Available at: http://www.ncbi.nlm.nih.gov/pubmed/19693780 [Accessed June 6, 2016].

Fedele, V. et al., 2011. Neurogenesis in the R6/2 mouse model of Huntington's disease is impaired at the level of NeuroD1. Neuroscience, 173, pp.76-81. Available at: http://www.sciencedirect.com/science/article/pii/S0306452210011292 [Accessed January 24, 2014].

Gil, J. et al., 2005. Reduced hippocampal neurogenesis in R6 / 2 transgenic Huntington 's disease mice. , 20, pp.744-751.

Giralt, A. et al., 2011. Increased PKA signaling disrupts recognition memory and spatial memory: role in Huntington's disease. Human molecular genetics, 20(21), pp.4232-47. Available at: http://www.ncbi.nlm.nih.gov/pubmed/21835884 [Accessed November 16, 2013].

Giralt, a et al., 2012. Long-term memory deficits in Huntington's disease are associated with reduced CBP histone acetylase activity. Human molecular genetics, 21(6), pp.1203-16. 
Available at: http://www.ncbi.nlm.nih.gov/pubmed/22116937 [Accessed November 21, 2013].

Gould, E. et al., 1999. Learning enhances adult neurogenesis in the hippocampal formation. Nature neuroscience, 2(3), pp.260-5. Available at:

http://www.ncbi.nlm.nih.gov/pubmed/10195219 [Accessed June 6, 2016].

Grote, H.E. et al., 2005. Cognitive disorders and neurogenesis deficits in Huntington's disease mice are rescued by fluoxetine. The European journal of neuroscience, 22(8), pp.2081-8. Available at: http://www.ncbi.nlm.nih.gov/pubmed/16262645 [Accessed November 1, 2013].

Ho, A.K. et al., 2003. Profile of cognitive progression in early Huntington's disease. Neurology, 61(12), pp.1702-1706. Available at: http://www.neurology.org/content/61/12/1702 [Accessed May 8, 2014].

Hult, S. et al., 2011. Mutant Huntingtin Causes Metabolic Imbalance by Disruption of Hypothalamic Neurocircuits. Cell Metabolism, 13(4), pp.428-439.

Kirkwood SC et al., 2001. Progression of symptoms in the early and middle stages of Huntington disease. Arch Neurol, 58, pp.273-8.

Kolodziejczyk, K. et al., 2014. Striatal Synaptic Dysfunction and Hippocampal Plasticity Deficits in the Hu97/18 Mouse Model of Huntington Disease. PLOS ONE, 9(4), pp.971983.

Krezymon, A. et al., 2013. Modifications of hippocampal circuits and early disruption of adult neurogenesis in the tg2576 mouse model of Alzheimer's disease. C. V. Borlongan, ed. PloS one, 8(9), p.e76497. Available at: http://dx.plos.org/10.1371/journal.pone.0076497 [Accessed May 14, 2014].

Kubik, S., Miyashita, T. \& Guzowski, J.F., 2007. Using immediate-early genes to map hippocampal subregional functions. Learning \& Memory, 14(11), pp.758-770.

Kung, V.W.S. et al., 2007. Dopamine-dependent long term potentiation in the dorsal striatum is reduced in the R6/2 mouse model of Huntington's disease. Neuroscience, 146(4), pp.1571-80. Available at: http://www.sciencedirect.com/science/article/pii/S0306452207003600 [Accessed January 24, 2014].

Lange, K.W. et al., 1995. Comparison of executive and visuospatial memory function in Huntington's disease and dementia of Alzheimer type matched for degree of dementia. Journal of neurology, neurosurgery, and psychiatry, 58(5), pp.598-606. Available at: http://www.pubmedcentral.nih.gov/articlerender.fcgi?artid=1073493\&tool=pmcentrez\&re ndertype=abstract [Accessed May 25, 2014].

Lawrence, A.D. et al., 2000. Visual object and visuospatial cognition in Huntington's disease: implications for information processing in corticostriatal circuits. Brain : a journal of 
neurology, 123 ( Pt 7, pp.1349-64. Available at:

http://www.ncbi.nlm.nih.gov/pubmed/10869048 [Accessed May 25, 2014].

Lazic, S.E. et al., 2004. Decreased hippocampal cell proliferation in R6 / 1 Huntington 's mice. Neuroreport, 15(5), pp.811-813.

Leuner, B. et al., 2004. Learning Enhances the Survival of New Neurons beyond the Time when the Hippocampus Is Required for Memory. Journal of Neuroscience, 24(34), pp.7477-7481. Available at:

http://www.jneurosci.org/cgi/doi/10.1523/JNEUROSCI.0204-04.2004 [Accessed June 6, 2016].

Lione, L.A. et al., 1999. Selective discrimination learning impairments in mice expressing the human Huntington's disease mutation. The Journal of neuroscience : the official journal of the Society for Neuroscience, 19(23), pp.10428-37. Available at: http://www.ncbi.nlm.nih.gov/pubmed/10575040 [Accessed May 26, 2014].

Lüesse, H.-G. et al., 2001. Evaluation of R6/2 HD transgenic mice for therapeutic studies in Huntington's disease: behavioral testing and impact of diabetes mellitus. Behavioural Brain Research, 126(1), pp.185-195.

Majerová, V. et al., 2012. Disturbance of real space navigation in moderately advanced but not in early Huntington's disease. Journal of the neurological sciences, 312(1-2), pp.86-91. Available at: http://www.ncbi.nlm.nih.gov/pubmed/21875725 [Accessed May $25,2014]$.

Montoya, A. et al., 2006. Episodic memory impairment in Huntington's disease: A metaanalysis. Neuropsychologia, 44(10), pp.1984-1994.

Murphy, K.P.S.J. et al., 2000. Abnormal Synaptic Plasticity and Impaired Spatial Cognition in Mice Transgenic for Exon 1 of the Human Huntington 's Disease Mutation. The Journal of Neuroscience, 20(13), pp.5115-5123.

Palfi, S. et al., 2007. Expression of mutated huntingtin fragment in the putamen is sufficient to produce abnormal movement in non-human primates. Molecular therapy : the journal of the American Society of Gene Therapy, 15(8), pp.1444-51. Available at: http://www.ncbi.nlm.nih.gov/pubmed/17505477 [Accessed May 13, 2016].

Paulsen, J.S. et al., 1995. Distinct cognitive profiles of cortical and subcortical dementia in advanced illness. Neurology, 45(5), pp.951-6. Available at: http://www.ncbi.nlm.nih.gov/pubmed/7746413 [Accessed May 23, 2016].

Petsophonsakul, P. et al., 2017. Memory formation orchestrates the wiring of adult-born hippocampal neurons into brain circuits. Brain Structure and Function, pp.1-17. Available at: http://link.springer.com/10.1007/s00429-016-1359-x [Accessed January 23, 2017].

Phillips, W., Morton, A.J. \& Barker, R.A., 2005. Abnormalities of Neurogenesis in the R6 / 2 
Mouse Model of Huntington 's Disease Are Attributable to the In Vivo Microenvironment. , 25(50), pp.11564-11576.

Richetin, K. et al., 2015. Genetic manipulation of adult-born hippocampal neurons rescues memory in a mouse model of Alzheimer's disease. Brain, 138(2), pp.440-455. Available at: https://academic.oup.com/brain/article-lookup/doi/10.1093/brain/awu354 [Accessed January 23, 2017].

Rosas, H.D. et al., 2003. Evidence for more widespread cerebral pathology in early HD: an MRI-based morphometric analysis. Neurology, 60(10), pp.1615-20. Available at: http://www.ncbi.nlm.nih.gov/pubmed/12771251 [Accessed July 10, 2014].

Simpson, J. et al., 2011. Altered adult hippocampal neurogenesis in the YAC128 transgenic mouse model of Huntington disease. Neurobiology of disease, 41(2), pp.249-60.

Available at: http://www.ncbi.nlm.nih.gov/pubmed/20875859 [Accessed May 25, 2014].

Snyder, J.S. et al., 2005. A role for adult neurogenesis in spatial long-term memory. Neuroscience, 130(4), pp.843-852.

Spargo, E., Everall, I.P. \& Lantos, P.L., 1993. Neuronal loss in the hippocampus in Huntington's disease: a comparison with HIV infection. Journal of Neurology, Neurosurgery \& Psychiatry, 56(5), pp.487-491. Available at: http://jnnp.bmj.com/cgi/doi/10.1136/jnnp.56.5.487 [Accessed April 22, 2016].

Sprengelmeyer, R. et al., 1995. Associative learning in degenerative neostriatal disorders: contrasts in explicit and implicit remembering between Parkinson's and Huntington's diseases. Movement disorders : official journal of the Movement Disorder Society, 10(1), pp.51-65. Available at: http://www.ncbi.nlm.nih.gov/pubmed/7885356 [Accessed May 23, 2016].

Stout, J.C. et al., 2011. Neurocognitive signs in prodromal Huntington disease. Neuropsychology, 25(1), pp.1-14. Available at: http://www.ncbi.nlm.nih.gov/pubmed/20919768 [Accessed May 3, 2016].

Verny, C. et al., 2007. Cognitive changes in asymptomatic carriers of the Huntington disease mutation gene. European Journal of Neurology, 14(12), pp.1344-1350. Available at: http://doi.wiley.com/10.1111/j.1468-1331.2007.01975.x [Accessed May 17, 2016].

Winner, B. et al., 2011. Adult neurogenesis and neurite outgrowth are impaired in LRRK2 G2019S mice. Neurobiology of Disease, 41(3), pp.706-716.

Winocur, G. et al., 2006. Inhibition of neurogenesis interferes with hippocampus-dependent memory function. Hippocampus, 16(3), pp.296-304. Available at: http://www.ncbi.nlm.nih.gov/pubmed/16411241 [Accessed July 20, 2014].

Zakzanis, K.K., 1998. The Subcortical Dementia of Huntington's Disease. Journal of Clinical and Experimental Neuropsychology (Neuropsychology, Development and Cognition: Section A), 20(4), pp.565-578. Available at: 
http://www.tandfonline.com/doi/abs/10.1076/jcen.20.4.565.1468 [Accessed May 23, 2016].

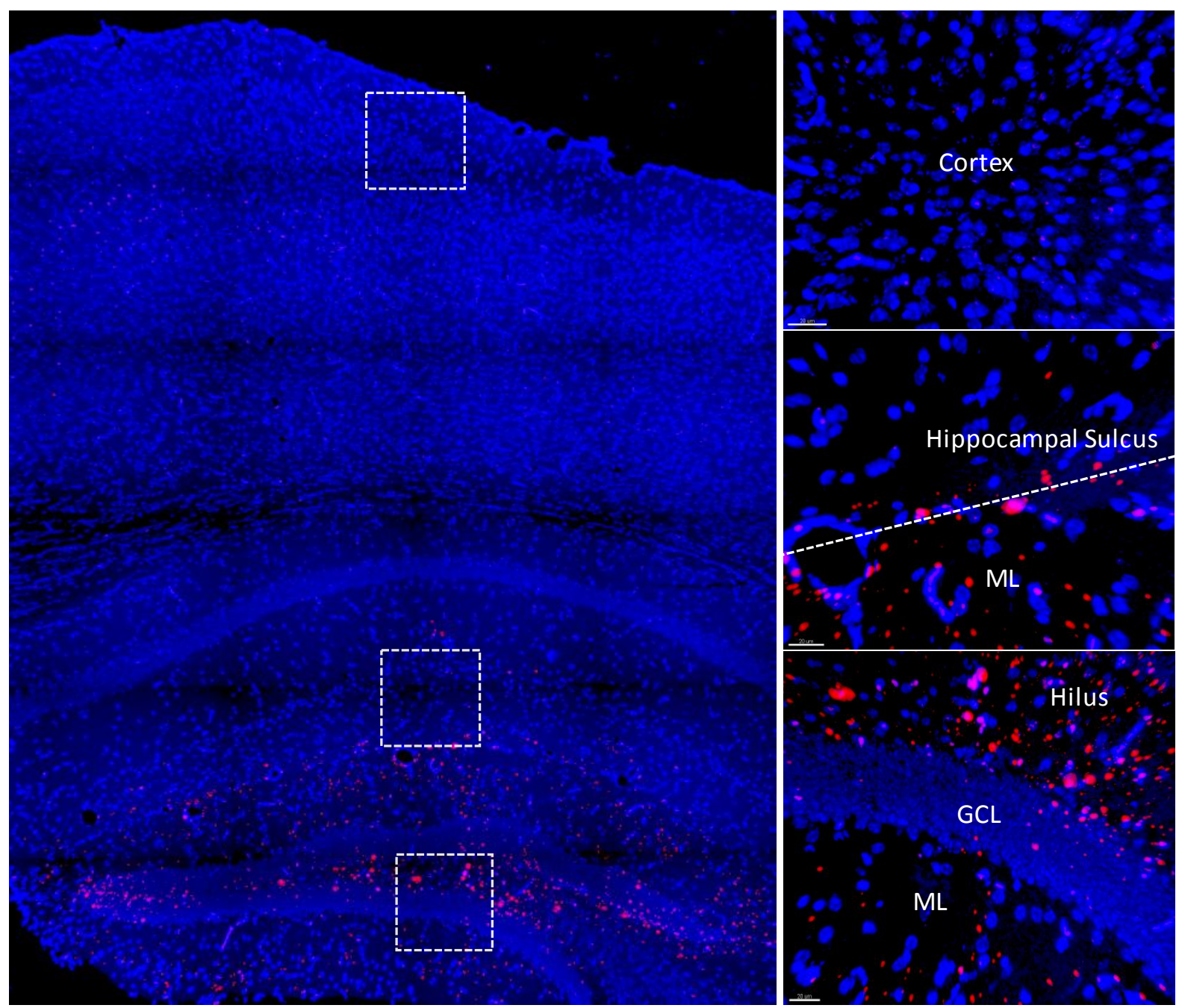

Figure S1: 12 weeks after injection of LV-mHTT, we observed formation of local mHTT aggregates in the molecular layer (ML), Granular Cell Layer (GCL), and Hilus of the hippocampus. However, very few aggregates were found in the upper hippocampal sulcus region and none in the adjacent cortex. 